\title{
Editorial
}

\section{Networked Dynamical Systems 2016}

\author{
Jinde Cao, ${ }^{1}$ Guoqiang Hu, ${ }^{2}$ Wei Lin, ${ }^{3}$ Guanghui Wen, ${ }^{1}$ Wenwu Yu, ${ }^{1}$ and Zhiqiang Zuo ${ }^{4}$ \\ ${ }^{1}$ Research Center for Complex Systems and Network Sciences, Department of Mathematics, Southeast University, Nanjing, China \\ ${ }^{2}$ School of Electrical and Electronic Engineering, Nanyang Technological University, Singapore \\ ${ }^{3}$ School of Mathematical Sciences, Fudan University, Shanghai, China \\ ${ }^{4}$ Department of Automation, Tianjin University, Tianjin, China
}

Correspondence should be addressed to Jinde Cao; jdcao@seu.edu.cn

Received 8 August 2016; Accepted 8 August 2016

Copyright ( $\odot 2016$ Jinde Cao et al. This is an open access article distributed under the Creative Commons Attribution License, which permits unrestricted use, distribution, and reproduction in any medium, provided the original work is properly cited.

Networked dynamical systems have been a hot topic in recent years due to their wide engineering applications in, for instance, attitude synchronization of spacecraft, design of distributed sensor networks, and scheduling of distributed microgrids. One common yet interesting phenomenon in the aforementioned systems is that various globally collective behaviors can emerge as a result of local interactions among neighboring individuals.

This special issue addresses new analysis and synthesis approaches for networked dynamical systems. It focuses not only on the networked dynamical systems' characteristics but also on the control strategies to achieve the desired collective behaviors. Call for papers has been carefully prepared by the guest editors and posted on the journal's web page, which has received much attention from researchers in various scientific communities. We have received 45 papers in these research fields. All manuscripts submitted to this special issue went through a thorough peer-refereeing process. Based on the reviewers' reports, 6 original research articles are finally accepted. The contents of this special issue contain modeling applications of complex networks, dynamical behavior analysis of complex networks, and synthesis and control of dynamical systems and networks. The contents are summarized as follows.

(1) Modeling and Applications of Complex Networks. In "P2P Lending Risk Contagion Analysis Based on a Complex Network Model" by Q. Wei and Q. Zhang, a complex network model of P2P lending risk contagion in China is constructed and general characteristics of direct risk contagion among China's online P2P lending platforms are simulated and analyzed in detail. It reveals that the superposition of media effects and information asymmetry is more likely to magnify the risk contagion effect.

(2) Dynamical Behavior Analysis of Complex Networks. "Attractor and Boundedness of Switched Stochastic CohenGrossberg Neural Networks" by C. Huang et al. studies the stochastic attractor and boundedness of a class of switched Cohen-Grossberg neural networks with discrete and infinitely distributed delays. New sufficient conditions regarding the mean-square uniformly ultimate boundedness, the existence of a stochastic attractor, and the mean-square exponential stability are established.

(3) Synthesis and Control of Dynamical Systems and Networks. The optimal regulation properties of multi-input and multioutput (MIMO) discrete-time networked control systems (NCSs), over additive white Gaussian noise (AWGN) fading channels, based on state-space representation, are investigated in "Optimal Tracking Performance of MIMO Discrete-Time Systems with Network Parameters" by C.-Y. Chen et al. The pinning controllability of directed complex delayed dynamical networks with periodic intermittent control scheme is investigated in "Pinning Controllability Scheme of Directed Complex Delayed Dynamical Networks via Periodically Intermittent Control" by S. Li et al. An algorithm is provided to determine the amounts of periodically intermittent controllers and locations of these intermittent controllers. "Robust Synchronization Controller Design for a Class of Uncertain Fractional Order Chaotic Systems” by L. Wang and C. Yang studies the synchronization problem for 
a class of uncertain fractional order chaotic systems. Combining the fractional order extension of the Lyapunov stability criterion and the proposed lemma, stability of the closed-loop system is analyzed, and two sufficient conditions to ensure the synchronization are also driven. A modified fractional order Chua chaotic circuit is proposed in "Stabilization of the Fractional-Order Chua Chaotic Circuit via the Caputo Derivative of a Single Input" by C. Yang et al. Two control schemes are designed to stabilize the modified fractional order Chua chaotic system via the Caputo derivative of a single input.

Note that the selected topics and papers are not a comprehensive representation of the area of the special issue. It is also worth noting that the published papers in this special issue do provide some recent advances in the field of networked dynamical systems, which could benefit the current research more or less.

\section{Acknowledgments}

We would like to thank the authors for their contributions. We also thank anonymous reviewers for their time and help in assessing the manuscripts.

Jinde Cao

Guoqiang $\mathrm{Hu}$

Wei Lin

Guanghui Wen

Wenwu Yu

Zhiqiang Zuo 


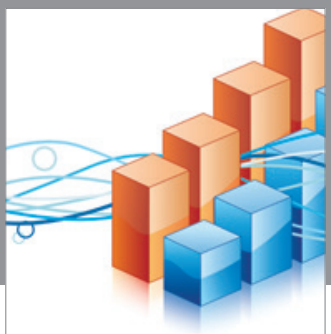

Advances in

Operations Research

vatem alat4

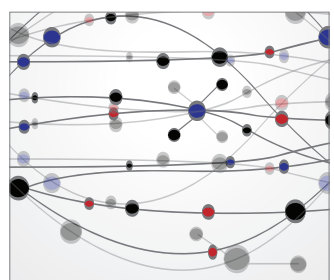

\section{The Scientific} World Journal
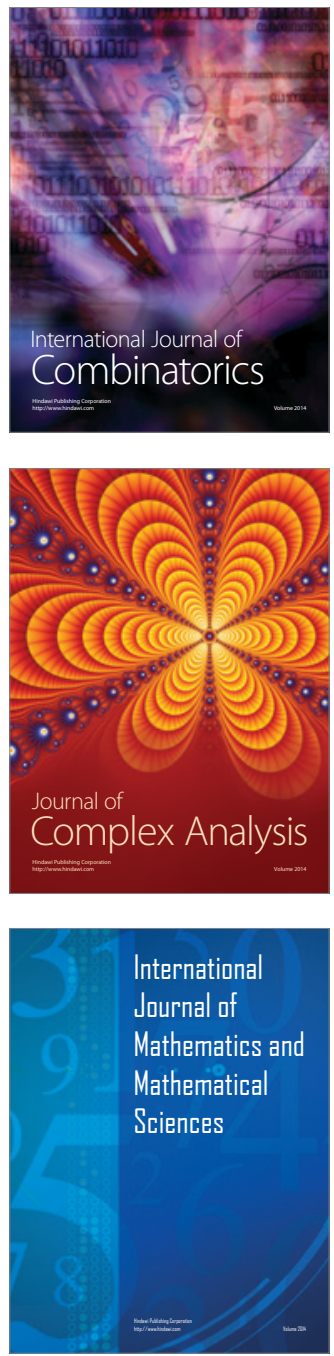
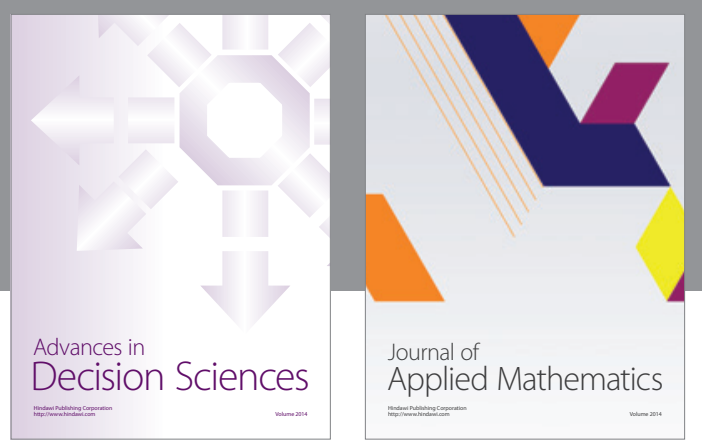

Algebra

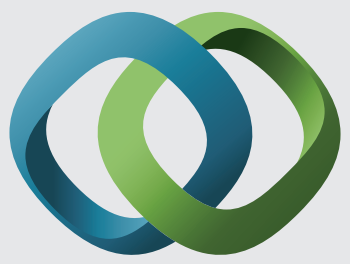

\section{Hindawi}

Submit your manuscripts at

http://www.hindawi.com
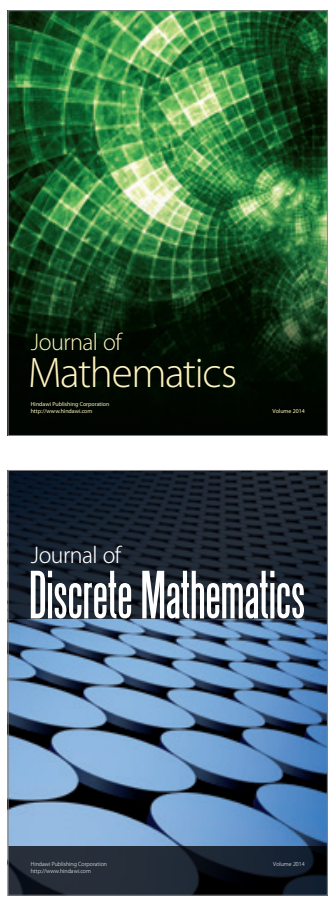

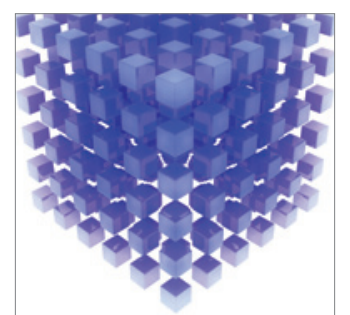

Mathematical Problems in Engineering
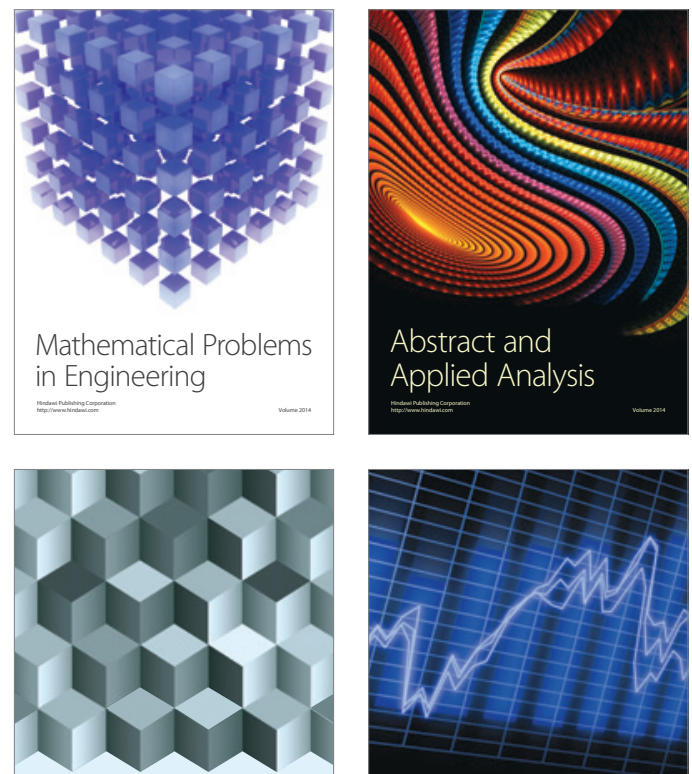

Journal of

Function Spaces

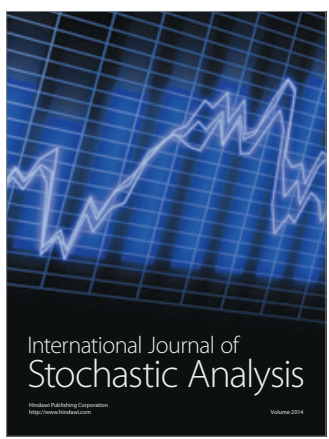

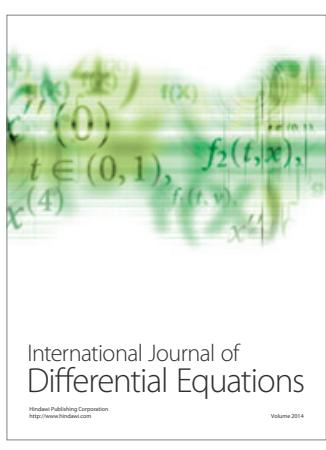
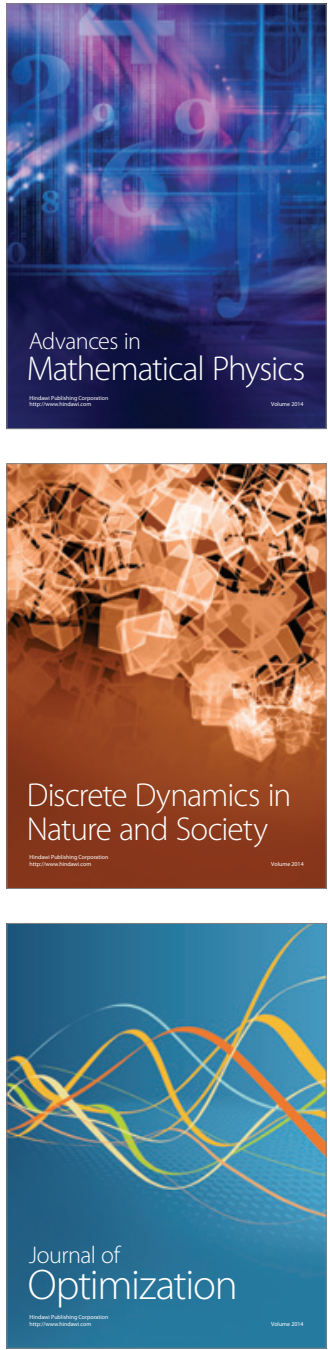